\title{
Percutaneous Endoscopic Lumbar Discectomy: Indications and Complications
}

Mingming Pan, MD, Qifan Li, MD, Sucheng Li, MD, Haiqing Mao, PhD, Bin Meng, PhD, Feng Zhou, PhD, and Huilin Yang, PhD

From: Department of Orthopaedic Surgery, The First Affiliated Hospital of Soochow University, Suzhou, Jiangsu Province, China

Address Correspondence: Bin Meng, PhD

Department of Orthopedics, the First Affiliated Hospital of Soochow University 899\# Pinghai Road, Suzhou, Jiangsu, PR China

E-mail: mbyang200o@126.com

Disclaimer: There was no external funding in the preparation of this manuscript. Conflict of interest: Each author certifies that he or she, or a member of his or her immediate

family, has no commercial association (i.e., consultancies, stock ownership, equity interest, patent/licensing arrangements, etc.) that might pose a conflict of interest in connection with the submitted manuscript.

Manuscript received: 02-05-2019 Revised manuscript received: 07-18-2019

Accepted for publication: 08-20-2019

Free full manuscript: www.painphysicianjournal.com
Background: Percutaneous endoscopic lumbar discectomy (PELD) often refers to percutaneous endoscopic transforaminal discectomy (PETD) and percutaneous endoscopic interlaminar discectomy (PEID). As a minimally invasive spinal procedure, PELD has gained increasing recognition for its small incision, quick recovery, short hospital stay, and equivalent clinical outcome compared to open surgery. In order to obtain satisfactory clinical efficacy, adequate consideration should be given regarding the indication of PELD. On the other hand, complications related to PELD will also significantly affect the safety and outcome of surgery.

Objective: Our objective was to conduct a literature review of the indications and complications of PELD and to provide our experience in patient selection and solutions to complications related to PELD.

Study Design: The study is a literature review focused on the indications and complications of PELD.

Setting: The study is a literature review on the indications and complications of PELD

Methods: A comprehensive review of available literature on PELD was performed. Particular focus was given to the development of indications and prevention of complications. The literature was searched in PubMed database, and key words were set as "percutaneous endoscopic lumbar discectomy", "percutaneous endoscopic transforaminal discectomy", "percutaneous endoscopic interlaminar discectomy", "PELD", "PETD", "PEID", "YESS" and "TESSYS".

Results: PELD is an effective and safe treatment for lumbar disc herniation, lumbar spinal stenosis, recurrent lumbar disc herniation, and other lumbar diseases. Complications related to PELD include dural tear, nerve root injury, recurrence, and so on.

Limitations: Some results drawn in this review are based on retrospective study or small sample size. Studies of larger sample size and more multicenter, randomized controlled trials should be conducted to evaluate the clinical efficacy and safety of PELD.

Conclusions: PELD is a promising surgical technique for lumbar diseases. Proper patient selection, excellent surgical skills, and rich experience are required for satisfactory outcomes.

Key words: Complications, indications, minimally invasive spine surgery, PELD

Pain Physician 2020: 23:49-56
0 ince Kambin (1) experimentally induced arthroscopy to treat lumbar disc herniation (LDH), percutaneous endoscopic lumbar discectomy (PELD) has undergone great development in the past decade. In 1997 and 2003, Yeung et al (2) and Hoogland et al (3) developed the Yeung endoscopic spine system (YESS) and transforaminal endoscopic spine system (TESSYS) techniques, respectively, and the 2 techniques are collectively named percutaneous endoscopic transforaminal discectomy (PETD). Afterwards, Ruetten 
(4) introduced percutaneous endoscopic interlaminar discectomy (PEID) in 2006. Both PETD and PEID have been proven to be equivalent to open spine surgery or other minimally invasive surgery in terms of efficacy and safety (5).

Indications of PELD ought to be taken into consideration for surgical efficacy and safety. With the development of surgical techniques, navigation techniques, and optical systems, indications of PELD have expanded from single LDH to lumbar spinal stenosis, lumbar metastatic tumor, lumbar discal cyst, and revision of recurrent LDH. On the other hand, surgery-related complications have become more common and inevitable due to the wide spread of PELD $(6,7)$. The most prevalent among them include dural tear, intervertebral infection, postoperative hypoesthesia, guidewire breakage, and recurrence of lumbar disc herniation. By the use of PubMed database and key words such as "percutaneous endoscopic lumbar discectomy", "percutaneous endoscopic transforaminal discectomy", "percutaneous endoscopic interlaminar discectomy", "PELD", "PETD", "PEID", "YESS" and "TESSYS", this study aims to summarize the indications of PELD, describe the complications, and explore their solutions.

\section{Percutaneous Endoscopic Transforaminal Discectomy}

In the YESS technique, the disc is accessed through the Kambin triangle approach, which consists of the exiting nerve root (hypotenuse), superior endplate of the caudal vertebra (base), and superior articular process of the caudal segment (height). The YESS procedure removes the nuclear pulposus with rongeur inside the annulus fibrosus, so TESS is an "inside-out" technology making it difficult to address migrated disc herniation. While using the TESSYS technique, a foraminoplasty is conducted by serial trephine and the endoscope is inserted into the spinal canal to deal with herniated disc materials. Therefore, TESSYS is an "outside-in" technology and has great advantage in treating highly migrated disc herniation and lumbar spinal stenosis. When it comes to the L5/S1 segment, puncture through the foramen can be technically difficult due to the high iliac crest, the large transverse process of $L 5$, and hidden disc materials, and in these cases an interlaminar approach may be a better choice $(8,9)$.

\section{Percutaneous Endoscopic Interlaminar DisceCTOMY}

Unlike PETD, PEID employs a posterior approach, passing through the lamina and ligamentum flavum to the disc, which is more familiar to spinal surgeons. Owing to the wide intervertebral space, PEID has advantages over PETD in treating disc herniation of L5/ $\mathrm{S} 1$. Besides, PEID can resect the ligamentum flavum and hypertrophy of the facet joint to resolve central stenosis and foraminal stenosis. As a significant complement to PETD, PEID is also suitable for highly migrated or calcified disc herniation because of the large operating space in the spinal canal (10-12). However, PEID requires traction of the thecal sac to deal with disc fragments, which may consequently cause dura laceration and other complications.

\section{Indications of Percutaneous Endoscopic LUMBaR DISCECTOMY}

\section{Lumbar Disc Herniation}

LDH has become more and more common because of the aging population and heavy work pressure (13). Symptomatic LDH should be addressed by surgical treatment if it is intractable for conservative treatment. Compared to open surgery, PELD has several superiorities. First, PELD has minimal trauma and blood loss. The incision of PELD is approximately $10 \mathrm{~mm}$ - just enough for the insertion of a working cannula, and blood loss is reported to be 5 to $51 \mathrm{~mL}$, which is significantly less than that for microendoscopic discectomy (MED) and open discectomy $(14,15)$. Benefiting from the minimal trauma, patients often experience a short hospital stay and early return to work after surgery (16-18). Second, PELD can be finished under local anesthesia or epidural anesthesia, so that safety is more reliable for the elderly in poor general condition. Besides, any damage to the nerve root can be detected from the instant feedback of patients, which enables surgeons to prevent this catastrophic complication (19-22). Third, differing from posterior open surgery, the working cannula used in PELD causes minimal damage to bony elements, preserving the stabilization of the spine and reducing the risk of secondary degenerative diseases. According to the anatomy of lumbar spine, the scale of the foramen decreases while the scale of the interlaminar space increases from L1 to L5 (23). In this situation, PETD is suitable for LDH in L3/4 or the upper index, while PEID is preferred in the $L 4 / 5$ and $L 5 / S 1$ levels.

\section{Lumbar Stenosis}

In patients with lumbar stenosis, the volume of spinal canal or foramen is decreased and the subsequent 
compression of the spinal cord or nerve root results in back pain, radiculopathy, and intermittent claudication. The etiology of lumbar stenosis consists of lumbar disc herniation, osteophytes of facet joints, and hypertrophy or ossification of soft tissue (posterior longitudinal ligament and ligament flavum). Based on the site of stenosis, lumbar stenosis is classified as central stenosis, lateral recess stenosis, or foraminal stenosis. Foraminoplasty in PETD is able to remove osteophyte and enlarge the scale of the foramen and lateral recess, so PETD is suitable for foraminal stenosis and lateral recess stenosis. Soft tissues and disc fragments can be resected via PEID with adequate operating space, so PEID is often applied to central and lateral recess stenosis (24-26).

\section{Lumbar Vertebral Metastasis}

Advanced malignant tumors including breast cancer, lung cancer, renal cell carcinoma, and prostate cancer may invade vertebrae, and then cause intractable pain and pathological vertebral fracture. Spinal canal encroachment also leads to compression of the spinal cord and nerve root. Considering the severity of advanced malignant tumors, the purpose of treatment often consists of relieving symptoms and improving quality of life. As a minimally invasive procedure, PELD has the ability to remove encroaching mass and decompress the spinal and nerve root with minimal trauma $(27,28)$. What is more, PELD can co-work with radiofrequency ablation to destroy invaded nerve roots so as to improve radiculalgia (29).

\section{Lumbar Discal Cyst}

Discal cyst is a rare lesion of the intervertebral disc. Unlike typical degenerative disc diseases (DDDs), sciatalgia $(75 \%)$ is the most common symptom of discal cyst, followed by low back pain (58\%). Signs of nerve root compression, including motor deficit (40\%) and hyposthesia (30\%), are predominant signs (30). Though spontaneous regression has been reported in some cases, most symptomatic discal cysts require surgical treatment. It is generally realized that discal cyst is related to DDDs, and PETD is able to resect cysts protruding into the spinal canal and repair the broken annulus effectively. Kim et al (31) first reported discal cyst cases addressed by PETD, in which postoperative radiograph showed nerve root decompression and symptoms were relieved significantly. Ha et al (32) also applied PETD to treat discal cyst in 8 patients. Scores for the Visual Analog Scale (VAS)-leg decreased from $8.25( \pm 0.5)$ to $2.25( \pm 2.21)$ and 4 cases were classified as excellent,
3 as good, and 1 as fair; therefore the rate of excellent or good was $87.5 \%$, according to Macnab criteria. However, the literature on lumbar discal cyst treated by PELD remains sparse, so studies of larger sample size are needed in the future.

\section{Recurrent Lumbar Disc Herniation}

Recurrent lumbar disc herniation is often identified as disc herniation occurring in the same segment 6 months after the primary surgery (33). Incidence of recurrence ranges from $2 \%$ to $18 \%$ according to different surgical options (34-36). Risk factors of LDH recurrence include male gender, older age ( $>50$ years), smoking habit, spinal trauma history, and central LDH (37). Scar tissue caused by primary surgery makes open revision harder as well as increases the risk of dural tear and nerve injury (38). In addition, posterior spinal stability is destroyed and secondary degenerative disc diseases are induced due to the resection of posterior lumbar structure once more $(39,40)$. Revision surgery through PELD keeps away from the scar tissue and prevents complications such as bleeding, dural tear, and nerve injury within a shorter operation time (41).

\section{Complications and Solutions of PELD}

\section{Dural Tear}

With PELD shifting from the "inside-out" technique to the "outside-in" technique, the incidence of dural tear has increased to $1.1 \%$. Damage to the dura mater by instruments or radiofrequency, adhesion in the spinal canal, giant disc fragments, and loosened dura are risk factors for dural tear. Most dural tears occur in the process of disc resection. Some lacerations can be recognized and repaired during the operation, while others may be neglected because of their small size, low volume of cerebrospinal fluid (CSF) leakage, and continuous intraoperative irrigation. PELD-related dural tear manifests in neurological deficit and intractable radiating pain aggravated by walking and change of position. Prevention of dural tear is of utmost importance. In patients with severe adhesion or other risk factors, caution should be taken during decompression. In PEID, excessive traction should be avoided to protect the thecal sac and nerve root. Small lacerations often heal spontaneously, and a horizontal position with adequate fluid infusion helps relieve some symptoms. With respect to giant laceration, open revision is traditionally required to repair the thecal sac and prevent further complications (42). Some researchers nowadays 
promote suture of the sac under endoscopy (43). As far as we are concerned, open revision is still a more proper option, since endoscopic repair needs excellent skill but its effect remains uncertain. For example, Tamaki et al (44) reported a lumbar disc herniating into the dura after PELD, which is a rare complication resulting from dural laceration and scar adhesion. The authors then carefully opened the dura, resected the herniated disc, and repaired the broken sac.

\section{Intervertebral Infection}

Incidence of intervertebral infection after spine surgery ranges from about $0.1 \%$ to $0.4 \%$; most cases are caused by bacterial infection (45). In a research study containing 209 cases of LDH treated by PETD, Gu et al (46) found one patient who was infected and recovered through intravenous antibiotics after 2 weeks, with the incidence being $0.47 \%$. The low trauma of PELD makes intervertebral infection uncommon, but the risk still exists. Pyogenic spondylodiscitis is a catastrophic complication after spine surgery, and it will cause serious dysfunction of the spinal nerve. Early examinations such as erythrocyte sedimentation rate (ESR) and C-reaction protein (CRP) should be conducted if there is any infection suspected. Magnetic resonance imaging (MRI) is of little value in early diagnosis. Needle biopsy of the disc guided by fluoroscopy is diagnostic and helpful in identifying pathogenic bacteria. Once diagnosed, patients with mild symptoms need positive antibiotics and a braking system on the bed. As for patients with severe symptoms and signs, intervertebral washing and drainage should be performed. Open debridement and fusion are necessary if conservative therapy is of no benefit.

\section{Postoperative Dysesthesia}

Postoperative dysesthesia (POD) is caused by irritation of instruments and improper operation. The dorsal root ganglion (DRG) lies in the intraforaminal region and is vulnerable to disc herniation, foraminal stenosis, and mechanical damage by operative instruments (47). Damage to the DRG brings about symptoms different from those associated with primary pathology. As a unique complication of PETD, POD greatly affects recovery and postoperative quality of life. Cho et al (48) applied the floating retraction technique (FRT) to prevent POD. The result revealed that no POD occurred in 154 patients, confirming that this technique was effective. Fluoroscopy is essential to locate guiding wire and working cannula so as to avoid mechanical stretch or damage to the upper DRG.

\section{Incomplete Removal of Disc}

Whether the resection of herniated disc is complete depends on the position of the working cannula, type of disc herniation, and size of herniated fragments. Choi et al (49) retrospectively analyzed 10,228 patients treated by PETD, and found 283 cases of incomplete resection, among which 95 were caused by improper location. With respect to the type of herniation, there were 91 cases with central herniation (32.2\%), 70 with migrated herniation (24.7\%), 63 with axillary type herniation $(22.3 \%), 18$ with shoulder-type herniation $(6.4 \%)$, and 12 with foraminal/extraforaminal herniation (4.2\%). Lee et al (50) found that herniations with high-canal compromise and high-grade migration make it harder for PELD to efficiently remove herniated disc. Herniated disc fragments ought to be adequately released from the annulus before they are grasped and removed. Detailed planning of the puncture route is the key to conducting a complete removal. A careful check for residual fragments is necessary, and putting the bevel of the working cannula towards fragments helps achieve sufficient removal of herniated disc. On the other hand, exceeding resection of the herniated disc may increase the risk of dural tear and damage to the nerve root, so surgeons just need to restore the normal motion and pulsation of the nerve root $(51,52)$.

\section{Recurrence of LDH}

The purpose of PELD is to remove herniated disc fragments and preserve normal nucleus pulposus. Therefore, recurrence of LDH sometimes occurs with aging, inappropriate weight-bearing, and other factors. PELD shares with other discectomy surgeries a number of risk factors for recurrence, like male gender, obesity (BMI $\geq 25$ ), old age ( $\geq 50$ years), trauma history, and central disc herniation. But PELD also has some unique risk factors for LDH recurrence?\}, such as surgeons' having less experience with PELD ( $\leq 200$ cases) and performing operations in the early develpment stage of PELD (before year 2010, considering the development of instruments and experience was incomplete) $(52,53)$. Preoperatively, surgeons should study imaging examinations and design the puncture route carefully. Postoperative instructions like lumbar muscle exercise, proper weight burden, and appropriate sitting posture are essential to decrease the possibility of LDH recurrence.

\section{Reoperation}

Causes of reoperation vary from residual fragments, LDH recurrence, discogenic back pain to discitis, 
and postoperative hematoma. In their retrospective study comparing PETD (group $A, n=301$ ) and microscopic discectomy (MD) (group $B, n=614$ ), Kim et al (54) found that 28 cases $(9.5 \%)$ in group $A$ and 38 cases $(6.3 \%)$ in group $B$ experienced reoperation, and the difference was of no significance. A study reported by Cheng et al (55) revealed that the incidence of reoperation in 6 months was greatest with PETD, followed by $\mathrm{MD}$, and lowest with open surgery $(P<.01)$; however, the incidence over 1 to 5 years was greatest with open surgery, followed by MD, and lowest with PETD $(P<$ $.01)$. The authors concluded that the long-term incidence of reoperation following PETD was relatively low. Minimally invasive transforaminal lumbar interbody fusion (Mis-TLIF), MED, and PETD are common surgery methods used for the revision of PELD. Yao et al (56) showed that the 3 methods share similar long-term surgical efficacy. Compared to Mis-TLIF, MED and PETD had the advantages of short operation time, hospital stay, and low expense, but the risk of recurrence was higher with these 2 surgery methods.

\section{Instrument Breakage}

Instrument breakage is uncommon with PELD, and just a few reports have been published. Despite its low incidence, this complication can be serious. Residual guiding wire can cause damage to vessels and nerves if it is not properly dealt with. Causes of breakage usually involve inappropriate puncture location or route, misoperation by surgeons, and poor quality or abrasion of the instrument. Guan et al (57) reported 2 cases of guiding wire fracture during PELD. The authors employed endoscopy to remove residual wires and continued to finish the surgery, and patients experienced great relief of symptoms after surgery without any significant complications. In order to avoid instrument breakage, care should be exercised in locating and puncturing - especially for the L5/S1 index. Broken guiding wire should be removed immediately when it breaks up. With the assistance of fluoroscopy, experienced surgeons can are able to remove the broken guidewire through endoscopy and a working cannula. For surgeons with little experience or intractable cases, open surgery is necessary.

\section{Conclusions and Outlook}

As a minimally invasive surgery, PELD is superior to conventional open surgery due to its shorter operation time, lower bleeding volume, minimal soft tissue damage, and faster return to work. However, it also has sev- eral disadvantages. First, surgeons are required to be very skilled in spine surgery. Incidence of complications or revisions for beginners could be high owing to the shortage of experience and proficiency, which results in a sheer learning curve $(58,59)$. Second, although local anesthesia applied in PELD helps protect nerve roots from damage, side effects could be greater with PELD compared to other procedures conducted by general anesthesia when patients cannot tolerate intraoperative pain. Severe painful feelings may even cause a deadly cardiovascular accident. Finally, frequent fluoroscopy is needed to obtain a correct puncture route, so radiation exposure to medical staff and patients is inevitable.

In terms of different surgery techniques, PETD and PEID each have their unique indications and complications. As we mentioned previously, PETD is more suitable in cases of L4/5 or the upper index. The PETD technique is also an appropriate treatment for lateral stenosis and foraminal stenosis. However, PETD has difficulty in dealing with highly migrated disc herniation, and in this situation incomplete removal of herniated disc fragments occurs. Improper location and puncturing in PETD can lead to injury of lumbar arteries and exiting nerve roots. Therefore, postoperative dysesthesia and hematoma are more common in PETD. On the other hand, PEID has advantages in L5/S1 lumbar disc herniation, central lumbar stenosis, and highly migrated disc herniation due to the great intervertebral space of the L5/S1 segment. However, excessive stretch in PEID can injure the thecal sac, so the risk of dural tear is higher than in PETD (60). In addition, local anesthesia is not applicable to PEID because of its relatively poor analgesic effect, which heightens the risks of anesthesia for elderly patients.

As for the operation, the following are some tips to be reminded of. Preoperatively, a detailed medical history and positive symptoms and signs ought to be recorded. Careful analysis of the imaging examination helps design the puncture route and avoid complications related to the instruments. Intraoperatively, surgeons should distinguish different anatomical structures under endoscopy, operate in a soft manner, and remember not to stretch nerve roots roughly. Nerve root function has to be monitored, and the operation must be stopped as soon as any damage occurs. Postoperatively, if the former symptoms remain or even worsen, surgeons should think about insufficient removal of compression. MRI is helpful for diagnosing and providing the proper 
solution. Patients with dizziness and headache may suffer CSF leakage, and can recover with horizontal recumbent positioning and adequate fluid infusion accompanied by a pressure dressing on the incision. Postoperative education about functional exercise is also important to stabilize the spine and reduce the possibility of LDH recurrence.

Due to the growing popularity of PELD, it has gained many developments and improvements. Fan et al (61) applied the isocentric navigation technique to assist puncture in difficult cases at the L5/S1 index for PETD; the "excellent" and "good" rating for patients" satisfaction reached $90.91 \%$. Li et al (62) developed a graded cannula system for foraminoplasty. The duck mouth-like cannulas shielded the exiting nerve root out of the working zone of trephine and protected the nerve root from being hurt. Among 134 cases followed up, there were 75 excellent cases, 49 good cases, and 5 fair cases, making the success rate $92.7 \%$.

PELD has been widely accepted by spinal surgeons owing to its satisfactory clinical safety and efficacy. More developments and techniques are expected to shorten the learning curve and promote its spread. We can conclude from the available literature that PELD is an effective and promising spine surgery procedure.

\section{References}

1. Kambin P. Arthroscopy microdiscectomy. Arthroscopy 1992; 8:287-295.

2. Yeung AT, Tsou PM. Posterolateral endoscopic excision for lumbar disc herniation: Surgical technique, outcome, and complications in 307 consecutive cases. Spine (Phila Pa 1976) 2002; 27:722-731.

3. Hoogland T, Schubert M, Miklitz B, Agnes R. Transforaminal posterolateral endoscopic discectomy with or without the combination of a low-dose chymopapain: A prospective randomized study in 280 consecutive cases. Spine (Phila Pa 1976) 2006; 31:E89o-E897.

4. Ruetten S, Komp M. A new full-endoscopic technique for the interlaminar operation of lumbar disc herniations using 6-mm endoscopes: Prospective 2-year results of 331 patients. Minim Invasive Neurosurg 2006; 49:80-87.

5. Ruan W, Feng F, Liu Z, Xie J, Cai L, Ping A. Comparison of percutaneous endoscopic lumbar discectomy versus open lumbar microdiscectomy for lumbar disc herniation: A meta-analysis. Int J Surg 2016; 31:86-92.

6. Ahn $\mathrm{Y}$, Lee $\mathrm{SH}$. Outcome predictors of percutaneous endoscopic lumbar discectomy and thermal annuloplasty for discogenic low back pain. Acta Neurochir (Wien) 2010; 152:1695-1702.

7. Kim DH, Choi G, Lee SH. Complications in percutaneous endoscopic lumbar diskectomy. In: Endoscopic Spine Procedures. New York, Thieme Medical
Publishers, Inc., 2011: pp. 253-267.

8. Ruetten S, Komp M, Merk H. Full-endoscopic interlaminar and transforamina lumbar discectomy versus conventional microsurgical technique: A prospective, randomized, controlled study. Spine (Phila Pa 1976) 2008; 33:931-939.

9. Nie H, Zeng J, Song $\mathrm{Y}$, et al. Percutaneous endoscopic lumbar discectomy for $\mathrm{L}_{5}$-S1 disc herniation via an interlaminar approach versus a transforaminal approach: A prospective randomized controlled study with 2-year follow up. Spine (Phila Pa 1976) 2016; 41:B30-B37.

10. Li ZZ, Hou SX, Shang WL, Song KR, Zhao HL. The strategy and early clinical outcome of full-endoscopic $\mathrm{L}_{5} / \mathrm{S}_{1}$ discectomy through interlaminar approach. Clin Neurol Neurosurg 2015; 133:40-45.

11. Dabo X, Ziqiang C, Yinchuan Z, et al. The clinical results of percutaneous endoscopic interlaminar discectomy (PEID) in the treatment of calcified lumbar disc herniation: A case-control study. Pain Physician 2016; 19:69-76.

12. Chen Y, Wang JX, Sun B, et al. Percutaneous endoscopic lumbar discectomy in treating calcified lumbar intervertebral disc herniation. World Neurosurg 2019; 122:el449-e1456.

13. Tian W, Lv Y, Liu Y, Xiao B, Han X. The high prevalence of symptomatic degenerative lumbar osteoarthritis in Chinese adults: A population-based study. Spine (Phila Pa 1976) 2014; 39:1301-1310.
14. Liu X, Yuan S, Tian Y, et al. Comparison of percutaneous endoscopic transforaminal discectomy, microendoscopic discectomy, and microdiscectomy for symptomatic lumbar disc herniation: Minimum 2-year follow-up results. J Neurosurg Spine 2018; 28:317-325.

15. Song HP, Sheng HF. A case-control study on the treatment of protrusion of lumbar intervertebral disc through PELD and MED. Exp Ther Med 2017; 14:3708-3712.

16. Choi G, Lee SH, Bhanot A, Raiturker PP, Chae YS. Percutaneous endoscopic discectomy for extraforaminal lumbar disc herniations: Extraforaminal targeted fragmentectomy technique using working channel endoscope. Spine (Phila Pa 1976) 2007; 32:E93-E99.

17. Lew SM, Mehalic TF, Fagone KL. Transforaminal percutaneous endoscopic discectomy in the treatment of far-lateral and foraminal lumbar disc herniations. J Neurosurg 2001; 94:216-220.

18. Sasani M, Ozer AF, Oktenoglu T, Canbulat N, Sarioglu AC. Percutaneous endoscopic discectomy for far lateral lumbar disc herniations: Prospective study and outcome of 66 patients. Minim Invasive Neurosurg 2007; 50:91-97.

19. Xu T, Tian R, Qiao P, Han Z, Shen Q, Jia $Y$. Application of continuous epidural anesthesia in transforaminal lumbar endoscopic surgery: A prospective randomized controlled trial. J Int Med Res 2019; 47:1146-1153. 
20. Zhu Y, Zhao Y, Fan G, et al. Comparison of the effects of local anesthesia and epidural anesthesia for percutaneous transforaminal endoscopic discectomy in elderly patients over 65 years old. Int J Surg 2017; 48:260-263.

21. Jia ZQ, He XJ, Zhao LT. Transforaminal endoscopic decompression for thoracic spinal stenosis under local anesthesia. Eur Spine J 2018; 27:465-471.

22. Choi G, Lee SH, Raiturker PP, Lee S, Chae YS. Percutaneous endoscopic interlaminar discectomy for intracanalicular disc herniations at $\mathrm{L}_{5}$-S1 using a rigid working channel endoscope. Neurosurgery 2006; 58:ONS59-ONS68.

23. Pait TG, Elias AJ. Thoracic, lumbar, and sacral spine anatomy for endoscopic surgery. Neurosurgery 2002; 51:S67-S78.

24. Yang JS, Chu L, Chen CM, et al. Foraminoplasty at the tip or base of the superior articular process for lateral recess stenosis in percutaneous endoscopic lumbar discectomy: A multicenter, retrospective, controlled study with 2-year followup. Biomed Res Int 2018; 2018:7692794

25. Lee $\mathrm{CH}$, Choi M, Ryu DS, et al. Efficacy and safety of full-endoscopic decompression via interlaminar approach for central or lateral recess spinal stenosis of the lumbar spine: A meta-analysis. Spine (Phila Pa 1976) 2018; 43:1756-1764.

26. Ahn Y. Percutaneous endoscopic decompression for lumbar spinal stenosis. Expert Rev Med Devices 2014; 11:605-616.

27. Joo YC, Ok WK, Baik SH, Kim HJ, Kwon OS, Kim KH. Removal of a vertebral metastatic tumor compressing the spinal nerve roots via a single-port, transforaminal, endoscopic approach under monitored anesthesia care. Pain Physician 2012; 15:297-302.

28. Tsai $\mathrm{SH}, \mathrm{Wu} \mathrm{HH}$, Cheng CY. Full endoscopic interlaminar approach for nerve root decompression of sacral metastatic tumor. World Neurosurg 2018; 112:57-63.

29. Wang D, Nie $Y$, Jiang DG. Reliving refractory pain of spinal metastasis patients with radiofrequency ablation through transforaminal endoscopy [in Chinese]. Zhonghua Yi Xue Za Zhi 2013; 93:2321-2323.

30. Aydin S, Abuzayed B, Yildirim H, Bozkus $\mathrm{H}$, Vural M. Discal cysts of the lumbar spine: Report of five cases and review of the literature. Eur Spine J 2010; 19:1621-1626.

31. Kim JS, Choi G, Lee CD, Lee SH. Removal of discal cyst using percutane- ous working channel endoscope via transforaminal route. Eur Spine J 2009; 18:201-205.

32. Ha SW, Ju Cl, Kim SW, Lee S, Kim YH, Kim HS. Clinical outcomes of percutaneous endoscopic surgery for lumbar discal cyst. J Korean Neurosurg Soc 2012; 51:208-214.

33. Lee JK, Amorosa L, Cho SK, Weidenbaum M, Kim Y. Recurrent lumbar disk herniation. J Am Acad Orthop Surg 2010; 18:327-337.

34. Guo JJ, Yang H. Long-term outcomes of the revision open lumbar discectomy by fenestration: A follow-up study of more than 10 years. Int Orthop 2009; 33:1341-1345

35. Moliterno JA, Knopman J, Parikh K, et al. Results and risk factors for recurrence following single-level tubular lumbar microdiscectomy. J Neurosurg Spine 2010; 12:680-686.

36. Shriver MF, Xie JJ, Tye EY, et al. Lumbar microdiscectomy complication rates: A systematic review and meta-analysis. Neurosurg Focus 2015; 39:E6.

37. Li Z, Yang H, Liu M, et al. Clinical characteristics and risk factors of recurrent lumbar disk herniation: A retrospective analysis of three hundred twentyone cases. Spine (Phila Pa 1976) 2018; 43:1463-1469.

38. Tafazal SI. Incidental durotomy in lumbar spine surgery: Incidence and management. Eur Spine J 2005; 14:287-290.

39. Le H, Sandhu FA, Fessler RG. Clinical outcomes after minimal-access surgery for recurrent lumbar disc herniation. Neurosurg Focus 2003; 15:1-4.

40. Cinotti G, Gumina S, Giannicola G Postacchini F. Contralateral recurrent lumbar disc herniation: Results of discectomy compared with those in primary herniation. Spine (Phila Pa 1976) 1999; 24:800-806.

41. Li X, Hu Z, Cui J, et al. Percutaneous endoscopic lumbar discectomy for recurrent lumbar disc herniation. Int J Surg 2016; 27:8-16.

42. Ahn Y, Lee HY, Lee SH, Lee JH. Dural tears in percutaneous endoscopic lumbar discectomy. Eur Spine J 2011; 20:58-64.

43. Shin JK, Youn MS, Seong YJ, Goh TS, Lee JS. latrogenic dural tear in endoscopic lumbar spinal surgery: Full endoscopic dural suture repair (Youn's technique). Eur Spine ] 2018; 27:544-548.

44. Tamaki Y, Sakai T, Miyagi R, et al. Intra- dural lumbar disc herniation after percutaneous endoscopic lumbar discectomy: Case report. J Neurosurg Spine 2015; 23:336-339.

45. Carragee EJ, Spinnickie AO, Alamin TF, Paragioudakis S. A prospective controlled study of limited versus subtotal posterior discectomy: Short-term outcomes in patients with herniated lumbar intervertebral discs and large posterior anular defect. Spine (Phila Pa 1976) 2006; 31:653-657.

46. Gu YT, Cui Z, Shao HW, Ye Y, Gu AQ. Percutaneous transforaminal endoscopic surgery (PTES) for symptomatic lumbar disc herniation: A surgical technique, outcome, and complications in 209 consecutive cases. J Orthop Surg Res 2017; 12:25.

47. Silav G, Arslan M, Comert A, et al. Relationship of dorsal root ganglion to intervertebral foramen in lumbar region: An anatomical study and review of literature. J Neurosurg Sci 2016; 60:339-344.

48. Cho JY, Lee SH, Lee HY. Prevention of development of postoperative dysesthesia in transforaminal percutaneous endoscopic lumbar discectomy for intracanalicular lumbar disc herniation: Floating retraction technique. Minim Invasive Neurosurg 2011; 54:214-218.

49. Choi KC, Lee JH, Kim JS, et al. Unsuccessful percutaneous endoscopic lumbar discectomy: A single-center experience of 10,228 cases. Neurosurgery 2015; 76:372-381.

50. Lee SH, Kang BU, Ahn Y, et al. Operative failure of percutaneous endoscopic lumbar discectomy: A radiologic analysis of 55 cases. Spine (Phila Pa 1976) 2006; 31:E285-E290.

51. Ahn Y. Transforaminal percutaneous endoscopic lumbar discectomy: Technical tips to prevent complications. Expert Rev Med Devices 2012; 9:361-366.

52. Yao $\mathrm{Y}$, Liu $\mathrm{H}$, Zhang $\mathrm{H}$, et al. Risk factors for recurrent herniation after percutaneous endoscopic lumbar discectomy. World Neurosurg 2017; 100:1-6.

53. Wang H, Zhou Y, Li C, Xiang L. Risk factors for failure of single-level percutaneous endoscopic lumbar discectomy. ] Neurosurg Spine 2015; 23:320-325.

54. Kim MJ, Lee SH, Jung ES, et al. Targeted percutaneous transforaminal endoscopic diskectomy in 295 patients: Comparison with results of microscopic diskectomy. Surg Neurol 2007; 68:623-631.

55. Cheng J, Wang H, Zheng W, et al. Reop- 
eration after lumbar disc surgery in two hundred and seven patients. Int Orthop 2013; 37:1511-1517.

56. Yao $\mathrm{Y}$, Zhang H, Wu J, et al. Comparison of three minimally invasive spine surgery methods for revision surgery for recurrent herniation after percutaneous endoscopic lumbar discectomy. World Neurosurg 2017; 100:641-647.

57. Guan X, Wu X, Fan G, et al. Endoscopic retrieval of a broken guidewire during spinal surgery. Pain Physician 2016; 19: E339-E342.

58. Ahn SS, Kim SH, Kim DW. Learning curve of percutaneous endoscopic lum- bar discectomy based on the period (early vs. late) and technique (in-and-out vs. in-and-out-and-in): A retrospective comparative study. J Korean Neurosurg Soc 2015; 58:539-546.

59. Kafadar A, Kahraman S, Akbörü M. Percutaneous endoscopic transforaminal lumbar discectomy: A critical appraisal. Minim Invasive Neurosurg 2006; 49:74-79.

6o. Chen J, Jing X, Li C, et al. Percutaneous endoscopic lumbar discectomy for $\mathrm{L}_{5} \mathrm{~S}_{1}$ lumbar disc herniation using a transforaminal approach versus an interlaminar approach: A systematic review and meta-analysis. World Neurosurg 2018; 116:412-420.

61. Fan G, Wang T, Hu S, Guan X, Gu X, He S. Isocentric navigation of percutaneous endoscopic transforaminal discectomy at the $L_{5} / S_{1}$ level in difficult puncture cases: A technical note. Pain Physician 2017; 20:E531-E540.

62. Li Z, Hou S, Shang W, Song K, Zhao H. New instrument for percutaneous posterolateral lumbar foraminoplasty: Case series of 134 with instrument design, surgical technique and outcomes. Int J Clin Exp Med 2015; 8:14672-14679. 